\title{
Theoretical Study on Sandwich-Like Transition-Metal- \\ Cyclooctatetraene Clusters and One-Dimensional Infinite Molecular Wires
}

Weicheng Gao, ${ }^{\mathrm{a}}$ XiaojingYao, ${ }^{\mathrm{b}}$ Yi Sun, ${ }^{\mathrm{a}}$ Weikang Sun, ${ }^{\mathrm{a}}$ Hongfei Liu, ${ }^{\mathrm{a}}$ Jianshuang Liu, ${ }^{\mathrm{a}}$ Yongjun Liu, ${ }^{\text {a }}$ Xiuyun Zhang ${ }^{a^{*}}$

${ }^{\mathrm{a} C o l l e g e}$ of Physics Science and Technology, Yangzhou University, No.180 Siwangting Road, Yangzhou 225002, China

${ }^{\mathrm{b} C o l l e g e}$ of Physics Science and Information Engineering, Hebei Normal University, No.20 Road East. 2nd Ring South, Yuhua District, Shijiazhuang 050024, China

Table S1. Structure parameters for $\mathrm{M}_{n}(\mathrm{COT})_{n+1}(\mathrm{n}=1,2)$ clusters and bimetal sandwich clusters as well as their infinite molecular wires. $\mathrm{R}_{\mathrm{C}-\mathrm{C}}$ and $\mathrm{R}_{\mathrm{C}-\mathrm{H}}$ are the length of $\mathrm{C}-\mathrm{C}$ and $\mathrm{C}-\mathrm{H}$ bonds of COT rings, and $\mathrm{R}_{\mathrm{M}-\mathrm{COT}}$ is the distance between transition metal atoms and the center of COT ring.

\begin{tabular}{c|c|c|c}
\hline system & $\mathrm{R}_{\mathrm{C}-\mathrm{C}}(\AA)$ & $\mathrm{R}_{\mathrm{C}-\mathrm{H}}(\AA)$ & $\mathrm{R}_{\mathrm{M}-\mathrm{COT}}(\AA)$ \\
\hline $\mathrm{Sc}(\mathrm{COT})_{2}$ & $1.40-1.43$ & 1.09 & $1.73,1.73$ \\
$\mathrm{Ti}(\mathrm{COT})_{2}$ & $1.39-1.43$ & 1.09 & $1.67,1.67$ \\
$\mathrm{Cr}(\mathrm{COT})_{2}$ & $1.38-1.44$ & $1.09-1.10$ & $1.41,2.13$ \\
$\mathrm{Mn}(\mathrm{COT})_{2}$ & $1.38-1.45$ & 1.09 & $1.74,1.74$
\end{tabular}




\begin{tabular}{c|c|c|c}
\hline $\mathrm{Sc}_{2}(\mathrm{COT})_{3}$ & $1.40-1.43$ & 1.09 & $1.64,1.78,1.78,1.64$ \\
$\mathrm{Ti}_{2}(\mathrm{COT})_{3}$ & 1.41 & $1.09-1.10$ & --- \\
$\mathrm{Cr}_{2}(\mathrm{COT})_{3}$ & $1.39-1.44$ & 1.09 & $1.37,2.28,2.28,1.37$ \\
$\mathrm{Mn}_{2}(\mathrm{COT})_{3}$ & 1.42 & 1.09 & $1.65,1.87,1.87,1.65$ \\
\hline$[\mathrm{Sc}(\mathrm{COT})]_{\infty}$ & 1.42 & 1.10 & 1.74 \\
{$[\mathrm{Ti}(\mathrm{COT})]_{\infty}$} & 1.41 & 1.10 & 1.73 \\
{$[\mathrm{Cr}(\mathrm{COT})]_{\infty}$} & 1.42 & 1.09 & 1.87 \\
{$[\mathrm{Mn}(\mathrm{COT})]_{\infty}$} & 1.42 & 1.09 & 1.87 \\
\hline
\end{tabular}

(a)

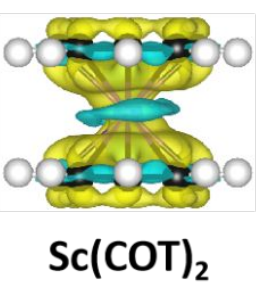

(e)

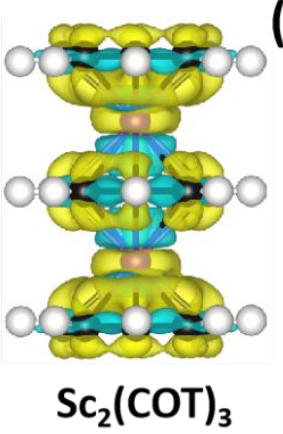

(b)

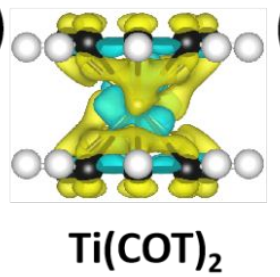

(f)

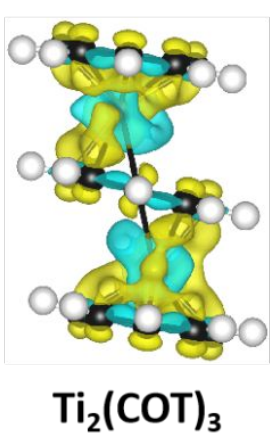

(c)

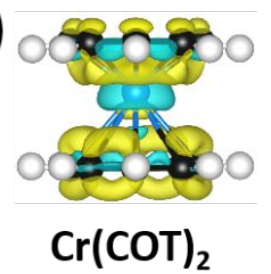

(g)

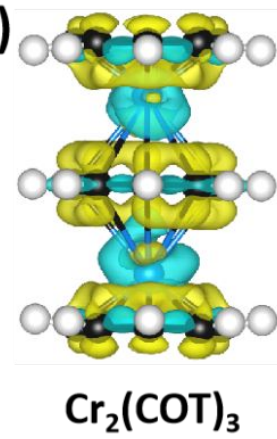

(d)

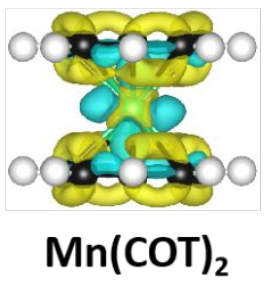

(h)

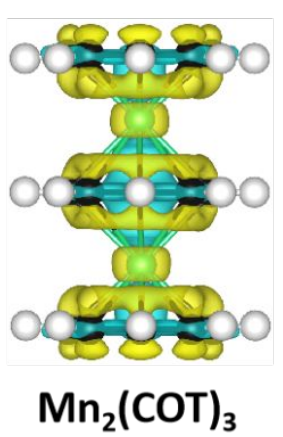

Figure S1. Charge density difference of $\mathrm{M}(\mathrm{COT})_{2}$ (a-d) and $\mathrm{M}_{2}(\mathrm{COT})_{3}$ (e-h) clusters. Yellow and blue colors refer to the electron accumulation and depletion, respectively. 
(a)

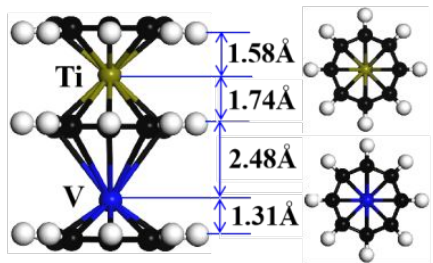

(COT) $\mathrm{V}(\mathrm{COT}) \mathrm{Ti}(\mathrm{COT})$

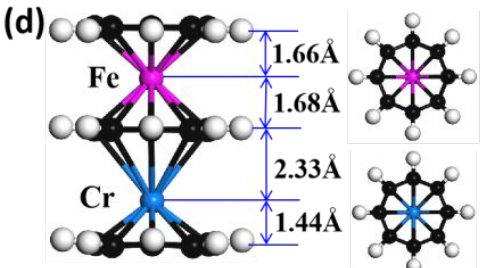

(COT) $\mathrm{Cr}(\mathrm{COT}) \mathrm{Fe}(\mathrm{COT})$

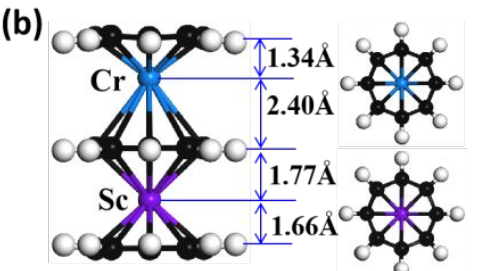

(COT) $\mathrm{Sc}(\mathrm{COT}) \mathrm{Cr}(\mathrm{COT})$

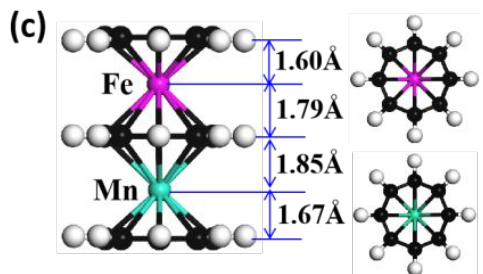

(COT)Mn(COT)Fe(COT)

(e)

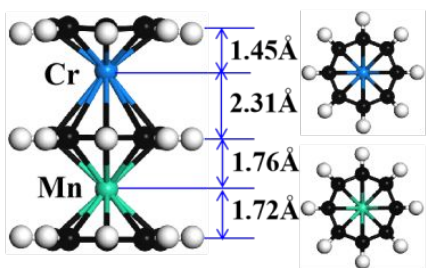

(COT) $\mathrm{Mn}(\mathrm{COT}) \mathrm{Cr}(\mathrm{COT})$

Figure S2. Side and top views of the optimized structures of mixed (COT) $\mathrm{M}_{1}(\mathrm{COT}) \mathrm{M}_{2}(\mathrm{COT})$ clusters, $\mathrm{M}=\mathrm{Ti}-\mathrm{Fe}(\mathrm{a}-\mathrm{e})$. 
Table S2. Structure parameters for bimetal sandwich clusters as well as their infinite molecular wires. $\mathrm{R}_{\mathrm{C}-\mathrm{C}}$ and $\mathrm{R}_{\mathrm{C}-\mathrm{H}}$ are the length of $\mathrm{C}-\mathrm{C}$ and $\mathrm{C}-\mathrm{H}$ bonds of COT rings, and $\mathrm{R}_{\mathrm{M}-\mathrm{COT}}$ is the distance between transition metal atoms and the center of COT ring.

\begin{tabular}{|c|c|c|c|}
\hline $\operatorname{System}\left[(\mathrm{COT}) \mathrm{M}_{1}(\mathrm{COT}) \mathrm{M}_{2(\mathrm{COT})}\right]$ & $\mathrm{R}_{\mathrm{C}-\mathrm{C}}(\AA)$ & $\mathrm{R}_{\mathrm{M} 1-\mathrm{COT}}(\AA)$ & $\mathrm{R}_{\mathrm{M} 2 \text {-СОT }}(\AA)$ \\
\hline $\mathrm{V}, \mathrm{Ti}$ & $1.40-1.43$ & $1.31,2.48$ & $1.58,1.74$ \\
\hline $\mathrm{Sc}, \mathrm{Cr}$ & $1.40-1.43$ & $1.66,1.77$ & $1.39,2.40$ \\
\hline $\mathrm{Cr}, \mathrm{Fe}$ & $1.41-1.42$ & $1.44,2.33$ & $1.66,1.68$ \\
\hline $\mathrm{Mn}, \mathrm{Fe}$ & $1.41-1.42$ & $1.67,1.85$ & $1.60,1.79$ \\
\hline $\mathrm{Cr}, \mathrm{Mn}$ & $1.41-1.42$ & $1.45,2.29$ & $1.72,1.76$ \\
\hline $\operatorname{System}\left(\left[(\mathrm{COT}) \mathrm{M}_{1}(\mathrm{COT}) \mathrm{M}_{2}\right]_{\infty}\right)$ & $\mathrm{R}_{\mathrm{C}-\mathrm{C}}(\AA)$ & $\mathrm{R}_{\mathrm{M} 1-\text { СОT }}(\AA)$ & $\mathrm{R}_{\mathrm{M} 2 \text {-СОТ }}(\AA)$ \\
\hline $\mathrm{V}, \mathrm{Ti}$ & $1.41-1.43$ & 1.88 & 1.77 \\
\hline $\mathrm{Sc}, \mathrm{Cr}$ & $1.40-1.42$ & 1.78 & 2.02 \\
\hline $\mathrm{Cr}, \mathrm{Fe}(-1 \mathrm{e})$ & $1.41-1.42$ & 1.96 & 1.83 \\
\hline $\mathrm{Mn}, \mathrm{Fe}(-1 \mathrm{e})$ & $1.40-1.42$ & 1.97 & 1.95 \\
\hline $\mathrm{Cr}, \mathrm{Mn}(-1 \mathrm{e})$ & $1.41-1.43$ & 2.00 & 1.88 \\
\hline
\end{tabular}

Table S3. Electron transferred from transition metal atoms to neighboring COT ligands for $(\mathrm{COT}) \mathrm{M}_{1}(\mathrm{COT}) \mathrm{M}_{2}(\mathrm{COT})$ clusters. $\mathrm{COT}(\mathrm{I})$ and $\mathrm{COT}(\mathrm{T})$ refers to the internal and terminal COT rings, respectively.

\begin{tabular}{c|c|l|l|c}
\hline$\left(\mathrm{M}_{1}, \mathrm{M}_{2}\right)$ & $\mathrm{M}_{1}>\operatorname{COT}(\mathrm{T})(\mathrm{e})$ & $\mathrm{M}_{1}>\operatorname{COT}(\mathrm{I})(\mathrm{e})$ & $\mathrm{M}_{2}>\operatorname{COT}(\mathrm{I})(\mathrm{e})$ & $\mathrm{M}_{2}>\operatorname{COT}(\mathrm{T})(\mathrm{e})$ \\
\hline
\end{tabular}




\begin{tabular}{c|c|c|c|c}
\hline $\mathrm{V}, \mathrm{Ti}$ & 0.90 & 0.20 & 0.69 & 0.71 \\
$\mathrm{Sc}, \mathrm{Cr}$ & 0.79 & 0.71 & 0.26 & 0.84 \\
$\mathrm{Cr}, \mathrm{Fe}$ & 0.87 & 0.23 & 0.47 & 0.53 \\
$\mathrm{Mn}, \mathrm{Fe}$ & 0.75 & 0.45 & 0.44 & 0.66 \\
$\mathrm{Cr}, \mathrm{Mn}$ & 0.84 & 0.26 & 0.58 & 0.62 \\
\hline
\end{tabular}

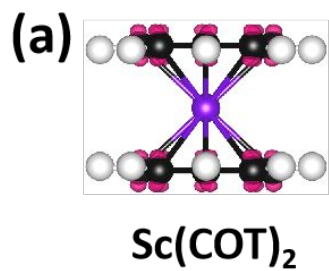

(e)

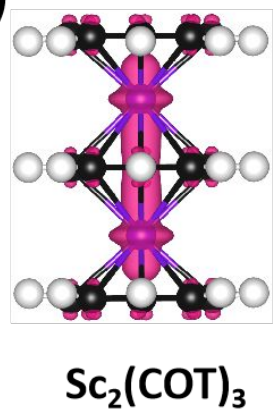

(b)

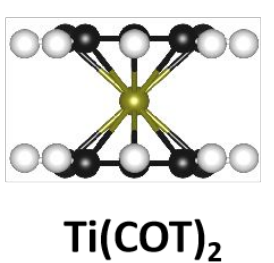

(f)

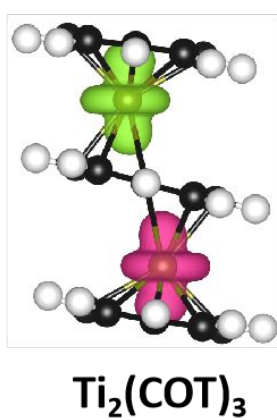

(c)

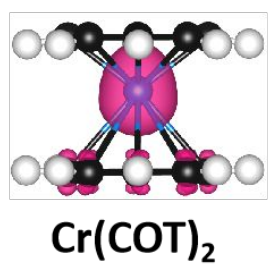

(g)

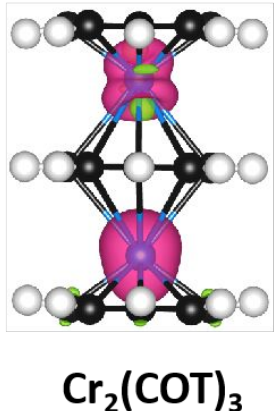

(d)

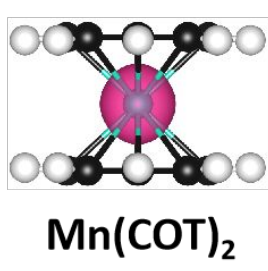

(h)

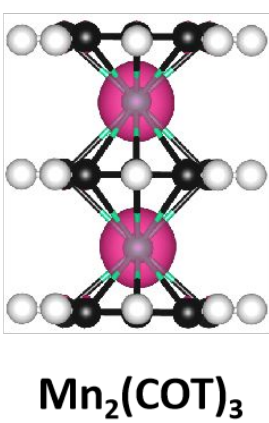

Figure S3. spin densities of $\mathrm{M}(\mathrm{COT})_{2}$ (a-d) and $\mathrm{M}_{2}(\mathrm{COT})_{3}$ (e-h) clusters, the red and green colors indicate spin-up and spin-down electron densities, respectively. 
(a)

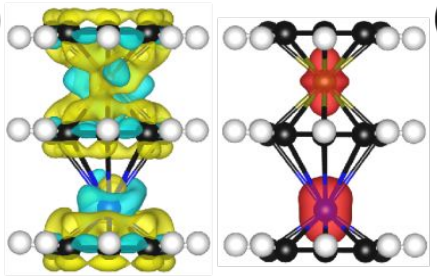

(COT)V(COT)Ti(COT)

(d)

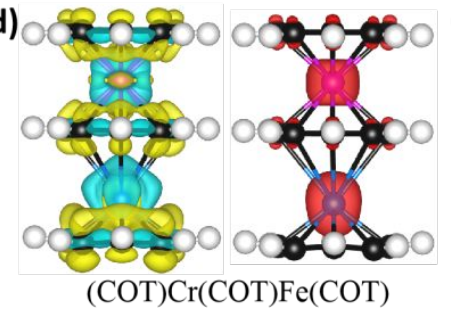

(b)

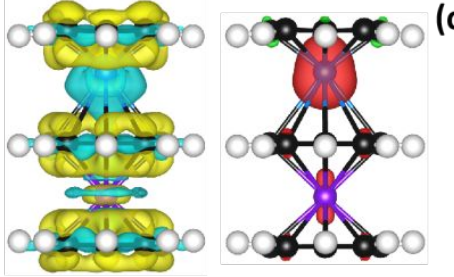

(COT) $\mathrm{Sc}(\mathrm{COT}) \mathrm{Cr}(\mathrm{COT})$

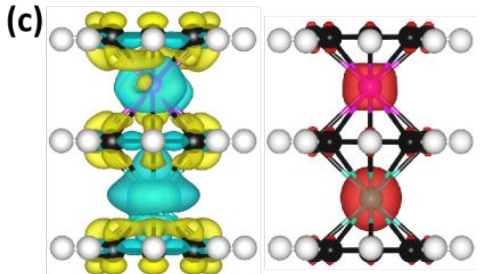

(COT)Mn(COT)Fe(COT)

(e)

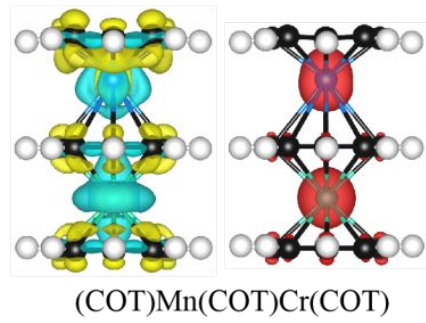

Figure S4. Charge density differences and spin densities for (a) (COT) $\mathrm{V}(\mathrm{COT}) \mathrm{Ti}(\mathrm{COT})$, (b) (COT) $\mathrm{Sc}(\mathrm{COT}) \mathrm{Cr}(\mathrm{COT}),(\mathrm{c})(\mathrm{COT}) \mathrm{Mn}(\mathrm{COT}) \mathrm{Fe}(\mathrm{COT}), \quad$ (d) (COT)Cr(COT)Fe(COT), and (e) (COT) $\mathrm{Mn}(\mathrm{COT}) \mathrm{Cr}(\mathrm{COT})$ cluster, respectively. The isosurface values for CDD and spin densities are $0.003 \mathrm{e} / \mathrm{Bohr}^{3}$ and $0.02 \mathrm{e} / \mathrm{Bohr}^{3}$, respectively. Blue and Yellow colors indicate electron depletion and accumulation, respectively. Red and green colors represent spin up and spin down densities, respectively.
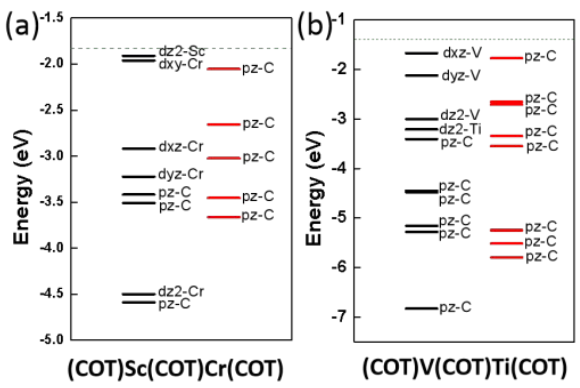
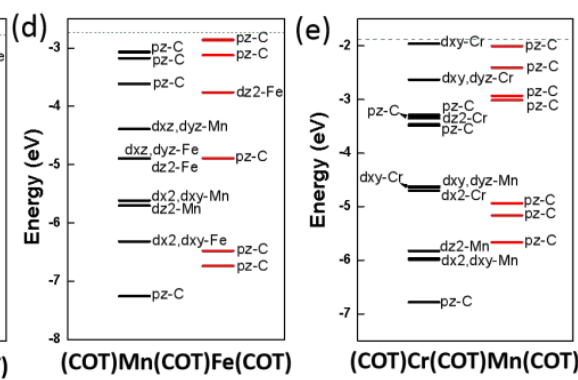

Figure S5. (a-e) Occupied orbitals for mixed metal clusters in two spins, black and red lines indicate spin up and spin down channels. 


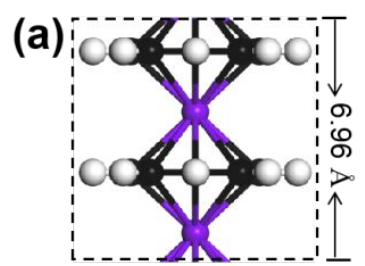

$[\mathrm{Sc}(\mathrm{COT})]_{\infty}$

(d)

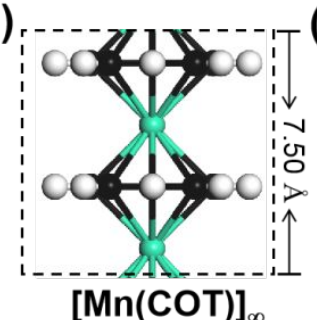

(g)

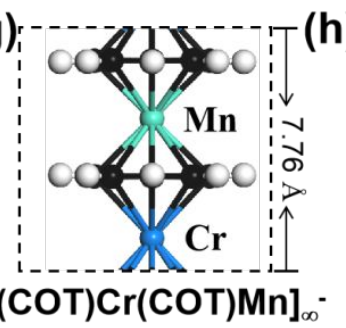

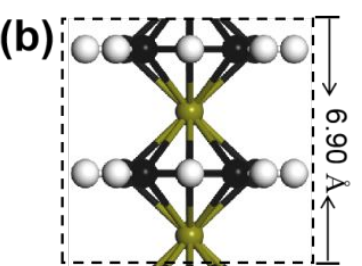

$[\mathrm{Ti}(\mathrm{COT})]_{\infty}$

(e)

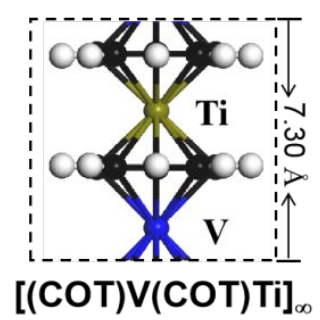

(c)

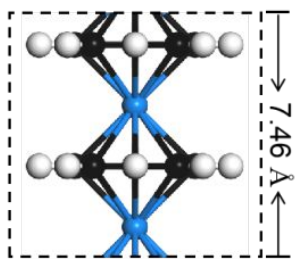

$[\mathrm{Cr}(\mathrm{COT})]_{\infty}$

(f)

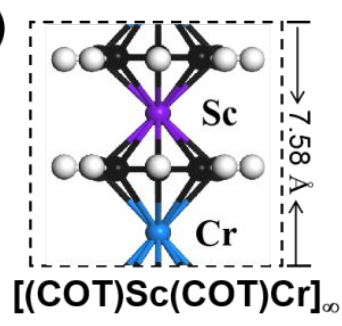

(i)
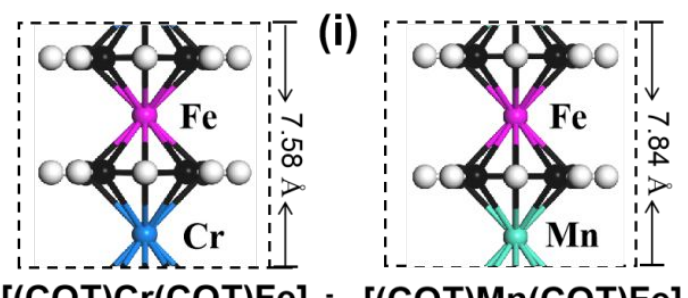

Figure S6. Optimized structures of $[\mathrm{M}(\mathrm{COT})]_{\infty}(\mathrm{a}-\mathrm{d})$ and $\left[(\mathrm{COT}) \mathrm{M}_{1}(\mathrm{COT}) \mathrm{M}_{2}\right]_{\infty}(\mathrm{e}, \mathrm{f})$ molecular wires. For $\left(\mathrm{M}_{1}, \mathrm{M}_{2}\right)=(\mathrm{Cr}, \mathrm{Fe}),(\mathrm{Mn}, \mathrm{Fe})$ and $(\mathrm{Cr}, \mathrm{Mn})$, the structures are after injecting one electron (g-i). 
Table S4.The binding energies $\left(\mathrm{E}_{\mathrm{b}}\right)$, magnetic moments (MM), band gaps $(\Delta)$, Bader charges the transition metal atoms (M_e) transferred to the COT rings and the charges every COT ring gained (COT_e) for $[\mathrm{M}(\mathrm{COT})]_{\infty}$.

\begin{tabular}{|c|c|c|c|c|c|c|}
\hline Sys & M & $\mathrm{E}_{\mathrm{b}}(\mathrm{eV})$ & $\operatorname{MM}\left(\mu_{B}\right)$ & $\Delta(\mathrm{eV})$ & M_e $(e)$ & COT_e (e) \\
\hline \multirow{4}{*}[\mathrm{M}(\mathrm{COT})]{$_{\infty}$} & $\mathrm{Sc}$ & 6.58 & 0 & 0 & 1.37 & 1.37 \\
\hline & $\mathrm{Ti}$ & 5.69 & 1.74 & 0 & 1.27 & 1.27 \\
\hline & $\mathrm{Cr}$ & 2.59 & 8 & 2.02 & 1.14 & 1.14 \\
\hline & $\mathrm{Mn}$ & 2.36 & 0 & 1.08 & 1.27 & 1.27 \\
\hline
\end{tabular}

